\title{
Visceral leishmaniasis, hypertriglyceridemia and secondary hemophagocytic lymphohistiocytosis
}

\author{
Claudia Colomba ${ }^{1}$ Paola Di Carlo ${ }^{1} \cdot$ Francesco Scarlata $^{1} \cdot$ Chiara Iaria $^{2}$. \\ Giuseppina Barberi $^{3} \cdot$ Fausto Famà $^{3} \cdot$ Valeria Cama $^{3} \cdot$ Antonio Cascio $^{1,4}$
}

Received: 27 December 2015 / Accepted: 2 February 2016 / Published online: 12 February 2016

(C) Springer-Verlag Berlin Heidelberg 2016

Dear Sir,

We found very interesting the paper by Lal et al. about hypertriglyceridemia as a possible diagnostic marker of disease severity in visceral leishmaniasis (VL) [1]. However, we think that authors should also have considered the possible diagnosis of secondary hemophagocytic lymphohistiocytosis (HLH).

HLH is a potentially fatal hyperinflammatory syndrome characterized by histiocyte proliferation and hemophagocytosis. HLH may be inherited (primary, familial) and occurs generally in infants or may be secondary to any severe infection, malignancy or rheumatologic condition and occurring in any age. HLH is diagnosed using clinical criteria developed by the HLH Study Group of the Histiocyte Society (Table 1) [2]. The mechanism behind secondary HLH is not well understood, its clinical picture may be identical to primary HLH, but there is more variability in severity and outcome. HLH is considered due to a deficiency in cytolytic activity resulting in persistent activation of lymphocytes and histiocytes. This uncontrolled immune response leads to hypersecretion of pro-inflammatory

Antonio Cascio

antonio.cascio03@unipa.it

1 Department of Health Promotion Sciences and Mother and Child Care "G. D' Alessandro", University of Palermo, Palermo, Italy

2 Infectious Diseases Unit-Azienda Ospedaliera PiemontePapardo, Messina, Italy

3 Department of Human Pathology, University of Messina, Messina, Italy

4 UOC Malattie Infettive e Centro Regionale di Riferimento AIDS-AOU Policlinico "P. Giaccone", Università di Palermo, Via del Vespro 129, 90127 Palermo, Italy cytokines, an upregulation of adhesion molecules and MHC I and II molecules on mono/macrophages, and an expansion of inflammatory monocytes. This exaggerated inflammatory response is responsible for necrosis and organ failure and results in uncontrolled proliferation and phagocytic activity of histiocytes [2].

Hypertriglyceridemia (fasting, greater than or equal to $265 \mathrm{mg} / 100 \mathrm{ml}$ ) is one of the current diagnostic criteria for HLH [2]. Several studies link hypertriglyceridemia to inhibition of lipoprotein lipase (LPL) by tumor necrosis factor- $\alpha(\mathrm{TNF}-\alpha)$, and TNF- $\alpha$ is a powerful autocrine and paracrine regulator of adipose tissue [3]. Indeed, many different sources of intense and prolonged T-lymphocyte/macrophage activation may be associated with dyslipidemia (particularly with hypertriglyceridemia) through inappropriate release of TNF-a, IFN-g, GM-CSF and respectively, of IL-1/IL-6, leading to adipose tissue lipolysis with increased VLDL secretion, decreased VLDL clearance, increased hepatic fatty acid synthesis, and suppression of fatty acid oxidation with HIV infection being the wellknown paradigm of this mechanism (possibly enhanced by some antiretroviral drugs).

In PubMed there are at least 70 papers in which the association leishmaniasis/(hemophagocytic or haemophagocytic) is present. Leishmania parasites have been found to be the most common protozoan trigger of acquired HLH. In a multicenter prospective study conducted to determine the frequency of HLH syndrome in children with VL, ten children out of $24(41 \%)$ with VL developed HLH syndrome [4]. HLH incidence in European adult population is about 1/800,000/year, with a reported prevalence of parasitic infections of $2.4 \%$ (53 out of 2197 subjects), and of Leishmania spp. of $0.77 \%$ (17 out of 2197) as a trigger. The clinical picture of VL with HLH initially can be indistinguishable from HLH of other etiology, potentially 
Table 1 HLH 2004 diagnostic criteria (modified from ref. 2)

The diagnosis of HLH can be established if one of either 1 or 2 below is fulfilled:

1. A molecular diagnosis consistent with HLH

2. Diagnostic criteria for HLH are fulfilled (five out of the eight criteria below):

\section{Fever}

Splenomegaly

Cytopenias (affecting $\geq 2$ lineages in the peripheral blood):

Hemoglobin $<90 \mathrm{~g} / \mathrm{l}$ (in infants $<4$ weeks): hemoglobin $<100 \mathrm{~g} / \mathrm{l}$

Platelets $<100 \times 10^{9} / 1$

Neutrophils $<1 \times 10^{9} / 1$

Hypertriglyceridemia and/or hypofibrinogenemia:

Fasting triglycerides $\geq 265 \mathrm{mg} / \mathrm{dl}$

Fibrinogen $\leq 1.5 \mathrm{~g} / \mathrm{l}$

Hemophagocytosis in bone marrow or spleen or lymph nodes

Low or absent NK-cell activity

Ferritin $\geq 500 \mu \mathrm{g} / \mathrm{l}$

Soluble CD25 $\geq 2400 \mathrm{U} / 1$

Comments:

(1) If hemophagocytic activity is not proven at the time of presentation, further search for hemophagocytic activity is encouraged. If the bone marrow specimen is not conclusive, material may be obtained from other organs. Serial marrow aspirates over time may also be helpful

(2) The following findings may provide strong supportive evidence for the diagnosis: (a) spinal fluid pleocytosis (mononuclear cells) and/or elevated spinal fluid protein, (b) histological picture in the liver resembling chronic persistent hepatitis (biopsy)

(3) Other abnormal clinical and laboratory findings consistent with the diagnosis are: cerebromeningeal symptoms, lymph node enlargement, jaundice, edema, skin rash. Hepatic enzyme abnormalities, hypoproteinemia, hyponatremia, VLDL $\uparrow$, HDL $\downarrow$

leading to misdiagnoses and inappropriate therapies [5]. However, hypergammaglobulinemia in a patient with HLH should raise suspicion for VL as potential trigger because this is a feature not commonly reported for other forms of HLH [5].

We think that HLH should be considered in severe cases of VL especially in those cases that do not promptly respond to specific treatment, and, on the contrary, the diagnosis of VL must be considered in children with HLH if they live or have travelled in VL endemic areas. The diagnosis of HLH must be pursued using the HLH 2004 diagnostic criteria (Table 1), while the diagnosis of VL must be pursued using appropriate and sensitive methods such as PCR testing of bone marrow specimens.

The preferred treatment for VL in the more economically developed countries is liposomal amphotericin B. Alternative medications are drugs containing pentavalent antimony, or miltefosine. The therapy of HLH includes steroids, cyclosporine A, etoposide, T-lymphocyte directed antibodies, and other biologicals; hematopoietic stem cell transplantation is the only way to cure hereditary HLH. Case reports and small series anecdotally have reported the successful use of liposomal amphotericin B or pentavalent antimony in VL complicated by HLH.

\section{Compliance with ethical standards}

Conflict of interest No conflict of interest to declare.

Funding There was not any financial support for this research.

Ethical approval Ethical approval was not required.

\section{References}

1. Lal CS, Verma RB, Verma N, et al. Hypertriglyceridemia: a possible diagnostic marker of disease severity in visceral leishmaniasis. Infection. 2015;44:39-45.

2. Gupta S, Weitzman S. Primary and secondary hemophagocytic lymphohistiocytosis: clinical features, pathogenesis and therapy. Expert Rev Clin Immunol. 2010;6:137-54.

3. Coppack SW. Pro-inflammatory cytokines and adipose tissue. Proc Nutr Soc. 2001;60:349-56.

4. Blazquez-Gamero D, Dominguez-Pinilla N, Chicharro C, et al. Hemophagocytic lymphohistiocytosis in children with visceral leishmaniasis. Pediatr Infect Dis J. 2015;34:667-9.

5. Bode SF, Bogdan C, Beutel K, et al. Hemophagocytic lymphohistiocytosis in imported pediatric visceral leishmaniasis in a nonendemic area. J Pediatr. 2014;165:147-153 e1. 